\title{
Comparison of clinical outcomes between open and modified endoscopic release for carpal tunnel syndrome
}

\author{
ZHONG CHEN $^{1}$, JUN LIU ${ }^{2}$, TANG-BO YUAN ${ }^{1}$, DA-WEI CAI ${ }^{1}$, XIAO-XU WANG ${ }^{3}$ and JIAN QIN $^{1}$ \\ ${ }^{1}$ Department of Orthopedics, Sir Run Run Hospital, Nanjing Medical University, Nanjing, Jiangsu 211100; \\ ${ }^{2}$ Department of Orthopedics, The Second Affiliated Hospital of Nanjing Medical University, Nanjing, Jiangsu 210000; \\ ${ }^{3}$ Department of Orthopedics, The Second Affiliated Hospital of Nanhua University, Hengyang, Hunan 421001, P.R. China
}

Received August 20, 2019; Accepted November 26, 2020

DOI: $10.3892 /$ etm.2021.10293

\begin{abstract}
The aim of the present study was to investigate a novel technology, requiring only a single portal and no special equipment, to perform endoscopic treatment of carpal tunnel (CT) syndrome (CTS). This novel technique involves a surgical approach and standard operating procedures and is designed to minimize the potential for complications. Patients with CTS were randomly assigned using a computer-generated random allocation and stratified by site to either the modified endoscopic CT release (MECTR) group $(n=48)$ or open CT release (OCTR) group $(n=46)$. Various medical indexes were compared between the two groups, including operative time, hospitalization time, the time required to resume a normal life or work, intraoperative complications, incision infection rate, the amelioration of symptoms (Kelly grading), post-operative scar pain score, recovery of grip strength and pinch strength, two-point discrimination and the presence of sympathetic dystrophy. The results revealed that all patients had grade A wound healing and the symptoms were completely relieved. No significant differences were observed between the two groups with regards to the incision infection rate, intraoperative complications, grip strength, pinch strength, two-point discrimination, presence of sympathetic dystrophy and clinical symptom amelioration. In addition, compared with the OCTR group, the MECTR group had a decreased operative and hospitalization time, post-operative scar pain score and time required to resume a normal lifestyle. Post-operative electromyographic examination also revealed that the median nerve sensory conduction speed increased compared with that prior to surgery in both groups. In conclusion, the use of MECTR for the treatment of CTS achieved higher patient satisfaction, a shorter operative time and hospitalization time,
\end{abstract}

Correspondence to: Professor Jian Qin, Department of Orthopedics, Sir Run Run Hospital, Nanjing Medical University, 109 Long Mian Avenue, Nanjing, Jiangsu 211100, P.R. China

E-mail: qinjian@njmu.edu.cn

Key words: carpal tunnel syndrome, median nerve, arthroscopy, modified, endoscopic an earlier return to work time or resumption of a normal life, as well as less post-operative scar pain compared with OCTR. Thus, these results suggested that MECTR may be an effective method for the treatment of idiopathic CTS. Trial registration no. ChiCTR2000041165, retrospectively registered 20th December 2020.

\section{Introduction}

Carpal tunnel (CT) syndrome (CTS), also known as delayed median nerve palsy, is one of the most common peripheral nerve entrapment syndromes diagnosed in the clinic (1). CTS frequently occurs in females aged 40-60 years and the male-to-female ratio is $1: 2-5$. The incidence rate in the US is $0.5-1.0 \%$ (2). Although no definite statistical data for CTS have been reported in China, it is clear that the morbidity rate is increasing with the improvement of living conditions. It may be hypothesized that carpal tunnel syndrome correlates with both an increase in the aging population and changing working conditions. The median nerve passes directly beneath the transverse carpal ligament and lies superficial to the nine flexor tendons of the digits, within close confinement of the CT; it is at this level that the median nerve is so easily compressed by any condition that increases the volume of the structures within the CT. The symptoms of median nerve compression were first described by Paget during the diagnosis and treatment of two patients with distal radius fractures in 1854, and CTS, a term first coined by Kremer, is the term currently used to describe the condition (3). The diagnosis and treatment of CTS has been approached from different perspectives and with different methods and there are currently several therapeutic methods available, ranging from non-surgical to surgical management. If non-surgical treatment is ineffective, surgery should be performed to alleviate the disease. The primary aim of the surgical treatment is to effectively remedy the compression state of the median nerve (4-6). To achieve this goal, surgical fasciotomy of the transverse carpal ligament and distal forearm are necessary (7). A number of different methods for surgical release have been reported, including traditional open dissection and double incision cannula $(8,9)$, a single small incision release (10) and endoscopic release (11). However, there remain several controversies regarding the advantages and disadvantages of the various surgical methods. 
Due to the rapid recovery and relatively small levels of inflicted trauma achieved with endoscopic CT release (ECTR), which was developed in Japan by Okutsu et al (12) in 1989, ECTR has been widely applied in the clinic. However, minimally invasive surgery via endoscopy requires expensive special equipment and the training required to perform the technique involves a long learning process; therefore, its application is restricted (13).

In recent years, the functionality of a modified endoscope for the treatment of CTS has been investigated. It is not the first time that ECTR through a single portal was described (14); however, in the novel method described in the present study, a small, single surgical incision was made without requiring the use of a special device. This method requires a probe, hook knife and ordinary arthroscopic systems, rather than the expensive facet endoscopic systems used in other procedures. In addition, the learning curve to use this method was discovered to be relatively short; therefore, this method may be easily learned by a new surgeon. In fact, the technique may be easily developed and practiced in areas with less advanced health systems. Due to the novel method achieving marked therapeutic results, a standardized operation procedure for the process is described in the present manuscript.

\section{Materials and methods}

Patients. The experimental protocol of the present study was approved by the Ethics Committee of Sir Run Run Hospital, Nanjing Medical University (Nanjing, China; approval no. 2015-SR-016) and was in accordance with the Declaration of Helsinki and institutional guidelines. A total of 94 patients aged 25-60 years with idiopathic CTS were randomized in the operating room into either an experimental or a control group. Factors that may have had a confounding effect (including age, gender, race, education level and living conditions) were stratified first, and then randomly grouped in each layer. Patients were seen by the same physician between January 2015 and March 2018. All patients had the typical symptoms in three and a half of the fingers on the radial side, including hypoesthesia, numbness, weakness in holding and no hyperalgesia (15). The patients had also received regular conservative treatment (e.g. oral non-steroidal anti-inflammatory drugs and rest) for $\geq 3$ months prior to the visit. Electromyography examination revealed that the sensory nerve conduction velocity had become slow and the motor conduction terminal latency was prolonged. The patients were randomly divided into the open CTR (OCTR) and modified ECTR (MECTR) groups. The OCTR group comprised 46 patients with a mean age of $48.0 \pm 5.33$ years and an average disease course of $10.6 \pm 2.87$ months. The MECTR group contained 48 cases, who had a mean age of $50.8 \pm 7.97$ years and an average disease course of $11.0 \pm 1.83$ months. In the OCTR group, the male to female ratio was 16:30. In MECTR group, the male to female ratio was 18:30. This ratio was not purposefully selected. Factors that may have had a confounding effect (including age, sex, race, education level and living conditions) were stratified first.

Diagnostic criteria. The diagnosis of CTS was considered in any patient who had pain, hypesthesia or paresthesia in the distribution of the median nerve in the hand or in any patient who had weakness or paralysis of the abductor pollicis brevis or opponens pollicis. In almost all cases, thenar atrophy was preceded by the onset of hypesthesia in the median nerve distribution following numerous months or years. In addition, the patients were continuously awakened from their sleep by burning pain and numbness in the thumb, as well as index and long fingers. The symptoms were alleviated through wrist activity.

Physical examination included the diagnosis of hypoesthesia in the affected fingers, atrophy of the thenar muscle innervated by the median nerve to varying degrees and a positive response to Tinel's sign and Phalen's maneuver. The results of the electromyogram, which is the preferred auxiliary examination for the diagnosis of median nerve compression, were required to indicate that the sensory latency of the distal median nerve was $\geq 3.5 \mathrm{msec}$ or $0.5 \mathrm{msec}$ higher than that on the contralateral side. Furthermore, the distal motor latency was required to be $\geq 4.5 \mathrm{msec}$ or exhibit a $\geq 1.0 \mathrm{msec}$ difference from the healthy side $(16,17)$.

Inclusion and exclusion criteria. The following inclusion criteria were applied: i) Typical symptoms (pain, hypesthesia or paresthesia) on one hand, including the thumb, index or middle finger, for $\geq 2$ weeks; ii) the symptoms were unrelieved, recurrent and even aggravated after regular non-surgical treatment for >3 months; iii) Hamada Grade I or II; iv) fulfillment of the electrodiagnostic criteria or if they were not fulfilled, the presence of night pain that awoke the patients and a positive flick test (shaking the wrist when the patients were asked what they do to relieve symptoms) were required.

The following exclusion criteria were used: i) Presence of secondary causes, such as bone abnormalities of the wrist, joint deformity or wrist occupations, including tumors or masses; ii) Hamada Grade III patients (sensory disturbances, severe thenar muscle atrophy and accompanying thumb-to-palmar dysfunction), as the effected thumbs usually required functional reconstruction; iii) diagnosis of inflammatory joint disease, trauma, diabetes mellitus or thyroid disorder to the affected hand during the preceding year, or pregnancy; iv) previous CTR surgery in the affected hand or CTR surgery in the contralateral hand during the preceding year; v) inability to complete questionnaires; and vi) evidence of diffuse peripheral neuropathy or cervical radiculopathy.

The use of all data from patients and their families was approved by the Sir Run Run Hospital ethics committee and written informed consent was obtained from each patient prior to surgery.

Surgical procedures. In the traditional OCTR group, the incision was made in the ulnar lateral of the thenar muscle crease, in order to avoid damaging the recurrent branch of the median nerve on the radial side of the wrist. The skin and subcutaneous tissue was incised to reveal the forearm and palm fascia using blunt separation and the incision of the fascia. The transverse carpal ligament was exposed and cut off along the ulnar lateral margin to avoid injuring the median nerve and recurrent branch. In the MECTR group, the incision was made to the proximal region of the transverse carpal crease and palmar longus tendon ulnar side. The length of the incision 
was $\sim 1 \mathrm{~cm}$, and it could be appropriately extended as required. The fascia was bluntly separated and longitudinally incised to determine the proximal edge of the transverse carpal ligament and the palmar longus tendon and to decrease the risk of damage. The ulnar bursa was separated on the ulnar side of the palmar longus tendon to distance it from the transverse carpal ligament. Then, the wrist joint was maintained in the dorsal position so that the nerve and tendons could be attached to the back of the CT. The distal skin of the incision was pulled up by a surgical hand hook and the transverse carpal ligament was exposed. In order to ensure complete release, the wrist joint of the patient was extended into extreme dorsiflexion during the operation, the palmar side of the carpal tunnel hooked from proximal to distal end 2-3 times, in order to confirm that no ligament tissue is hooked. Ophthalmic scissors were used to create the greatest possible longitudinal incision of the ligament under direct vision. Subsequently, a $4.0 \mathrm{~mm}$ in diameter, $30^{\circ}$ wide-angle common arthroscope lens without a metal sleeve was implanted beneath the distal transverse carpal ligament. The skin was pulled upward onto the palmar skin of the endoscopic channel by a suspension suture with 1 or 2 stitches, without using water perfusion. Once the smooth and white transverse carpal ligament was visualized through the microscope, a hook knife was inserted in front of the arthroscope to cut it off completely. Finally, the incision was sutured and pressure-dressed prior to removing the tourniquet. Intraoperative photographs are presented in Figs. 1-5. Fig. 1 is an intraoperative photo highlighting that in traditional incision surgery, an $\sim 6.0 \mathrm{~cm}$ long $\mathrm{S}$-shaped incision is made on the ulnar side of the thenar muscle crease. Fig. 2 is an intraoperative photo illustrating how the hand surgical hook is used to incise the transverse carpal ligament. Fig. 3 is an intraoperative photo demonstrating that after the transverse carpal ligament is incised completely, the median nerve is exposed. Fig. 4 is an intraoperative photo indicating that a $4 \mathrm{~mm}, 30^{\circ}$ wide-angle common knee joint arthroscope lens without a metal sleeve was implanted beneath the distal transverse carpal ligament. Fig. 5 demonstrates that the incision is sutured after the surgery. Fig. 6 is the surgical approach diagram of the modified endoscope before surgery.

Post-operative treatment. Following surgery in the OCTR group, the forearm was immobilized with plaster to maintain the wrist joint in the functional position for 1 week. The plaster was subsequently removed and the patients were encouraged to exercise the wrist. The patients in the MECTR group were encouraged to exercise the wrist joint $24 \mathrm{~h}$ after surgery, without immobilization.

Observational indicators. Evaluations were performed the week prior to surgery and again at 4, 8, 12 and 52 weeks post-surgery, in addition to 4 years after surgery, with an average follow-up time of 33.6 months. In this extended follow-up, the observations included surgical operative time, hospitalization time, intraoperative secondary injury rate (the incidence of nerve, vessel and tendon injury), incision infection rate, post-operative scar pain score, the time required to resume a normal lifestyle, recovery of grip and pinch force, two-point discrimination 3 months following the operation, the symptom of sympathetic dystrophy and symptom amelioration (Kelly grading).

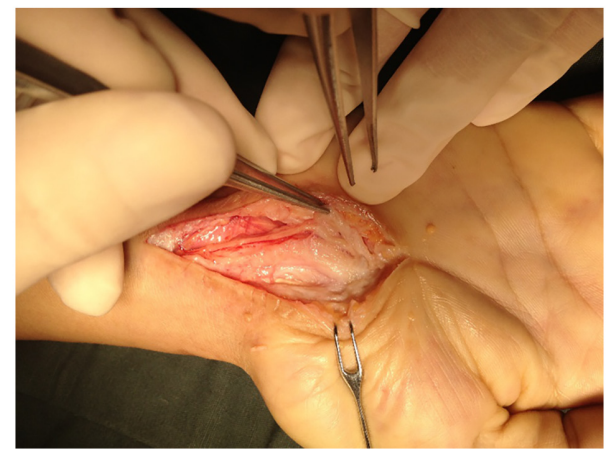

Figure 1. Intraoperative photograph of traditional incision surgery. An $\sim 6-\mathrm{cm}$ long S-shaped incision was made on the ulnar side of the thenar muscle crease. This is a demonstration of the open release surgical approach.

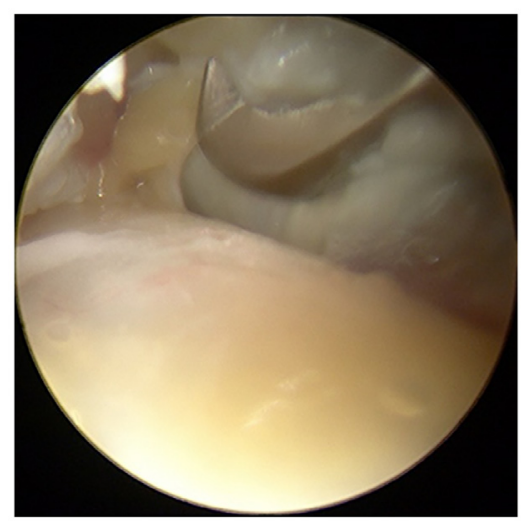

Figure 2. Intraoperative photograph of carpal ligament incision. A surgical hand hook was used to incise the transverse carpal ligament. This is a demonstration of the modified endoscopic release surgical approach.

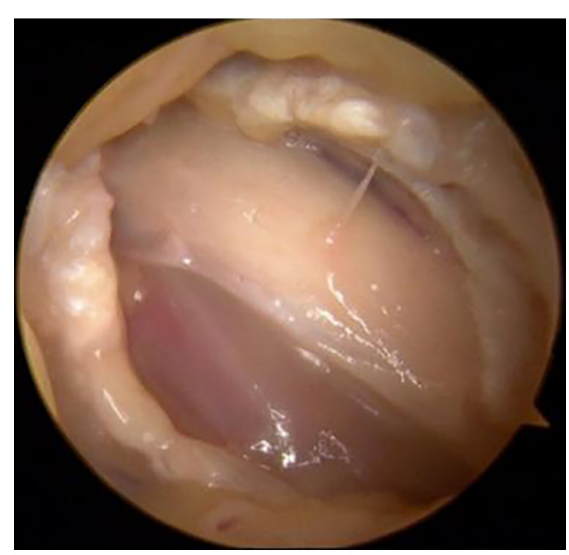

Figure 3. Intraoperative photograph of median nerve exposure. After the transverse carpal ligament was completely incised, the median nerve was exposed. This is a demonstration of the modified endoscopic release surgical approach.

Post-operative efficacy evaluation. The clinical symptom relief criteria were assessed according to the Kelly grading system (18). The differences in treatment efficacy were evaluated in a semi-quantitative manner (19). Semi-quantitative evaluation was performed by rating the outcomes using certain grades to obtain ranked data, e.g. regarding clinical efficacy, cases were rated as cured, markedly effective, improved and 


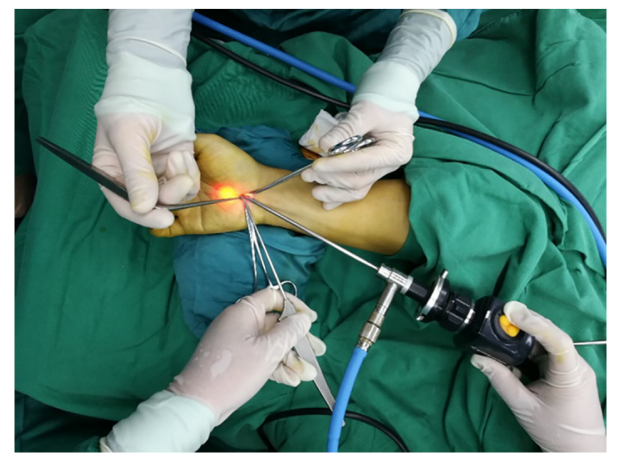

Figure 4. Intraoperative photograph of arthroscope implantation. A $4 \mathrm{~mm}$, $30^{\circ}$ wide-angle common knee joint arthroscope lens without a metal sleeve was implanted beneath the distal transverse carpal ligament. This is a demonstration of the modified endoscopic release surgical approach.

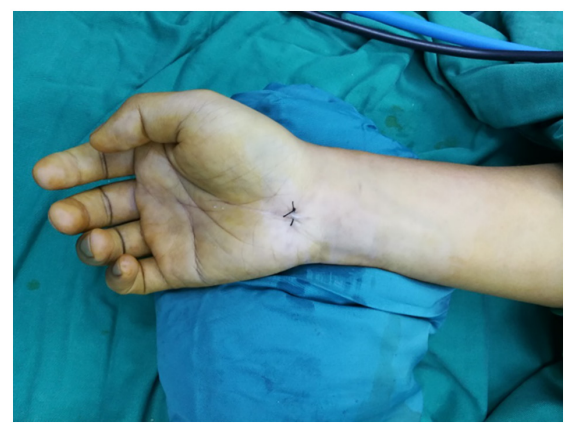

Figure 5. Intraoperative photograph. The incision was sutured following the surgery. The $1 \mathrm{~cm}$ incision made was located at the proximal end of the transverse carpal crease and palmar longus tendon ulnar side. This is a demonstration of the modified endoscopic release surgical approach.

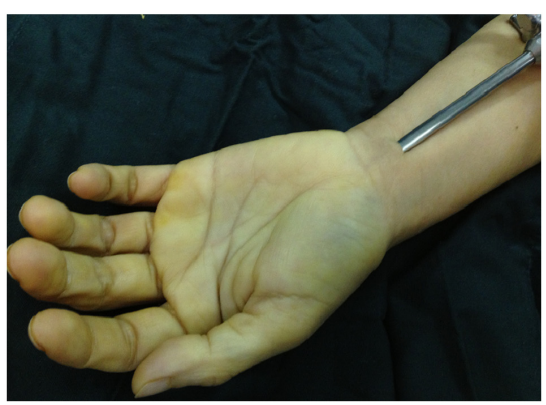

Figure 6. Incision in the modified endoscopic release surgical approach. The $1 \mathrm{~cm}$ incision was located in the proximal region of the transverse carpal crease and palmar longus tendon ulnar side.

invalid clinical test. Clinical tests included wound healing grade, incision infection rate, intraoperative complications, grip and pinch force, two-point discrimination, symptoms of sympathetic dystrophy and clinical symptom amelioration (Kelly). These tests were all performed by the same physician. Clinical efficacy was divided into cured, markedly effective, improved and invalid; clinical test results were divided into ,,,-++++++ , and the severity of pain symptoms is divided using 0-10 points for different levels of pain, 0 for no pain, 10 for severe pain. 0: No pain; 1-3 points: Mild pain, normal life, no sleep disturbance; 4-6 points: Obvious pain, unbearable,
Table I. Distribution of Hamada grades among the patients.

\begin{tabular}{lccc}
\hline Group & I & II & III \\
\hline OCTR $(n=46)$ & 22 & 24 & 0 \\
MECTR $(n=48)$ & 20 & 28 & 0 \\
Z & & -0.42 & \\
P-value & & 0.674 & \\
\hline
\end{tabular}

Patients with Hamada Grades I and II were considered for the OCTR and MECTR group, while those with Hamada Grade III were excluded, as these patients have severe thenar muscle atrophy and require functional revascularization. MECTR, modified endoscopic carpal tunnel release; OCTR, open carpal tunnel release.

analgesic drugs required, sleep disturbed; 7-10 points: Severe pain, intolerable, need analgesic, sleep by serious interference, can be accompanied by autonomic nervous disorder or passive position (20). The Kelly grading system used was as follows: i) Excellent-following the operation, the pain and numbness of the affected fingers had disappeared completely, the grip strength of the fingers, thumb-to-palmar activity and the range of joint activity had all returned to normal and the patient had resumed their daily work, with no recurrences observed following the operation; ii) good-symptoms were mostly relieved, with only a few symptoms still remaining, the grip and pinch force had returned to $3 / 4$ of the normal level and the patients were able to engage in light work; iii) general-symptoms were only slightly improved and most symptoms persisted, with the grip and pinch strength being only $\leq 1 / 2$ of that of normal; and iv) bad-the symptoms had not improved or in certain circumstances, the symptoms were even aggravated and the patient was required to change jobs or give up work. The rate of excellent and good Kelly grading was calculated as follows: Rate $(\%)=\left(\mathrm{n}_{\text {excellent }}+\mathrm{n}_{\text {good }}\right) / \mathrm{n}_{\text {total cases }}$ x100.

Statistical analysis. SPSS 13.0 statistical software (SPSS, Inc.) was used for statistical analysis. Count data were expressed as $\mathrm{n}(\%)$ and analyzed using a $\chi^{2}$ test. Continuous variables were expressed as the mean \pm standard deviation and the statistical significance of differences was analyzed using a t-test. The rank data were analyzed using a Wilcoxon rank-sum test. $\mathrm{P}<0.01$ was considered to indicate a statistically significant difference.

\section{Results}

Patient characteristics. The data, including sex, age, surgical side, duration of pre-operative symptoms and mean follow-up time in the two groups were comparable $(\mathrm{P}>0.05)$. The severity of the disease in each group was assessed using the Hamada classification (21-23) standard as follows: i) Grade I, numbness present in the radial side of the hand; ii) Grade II, in addition to Grade I, greater thenar muscle atrophy was also observed; and iii) Grade III, in addition to Grade II, thumb-to-palm dysfunction was also observed. The present study excluded Hamada Grade III patients, as these patients have severe 
Table II. Basic information of the two groups.

\begin{tabular}{lccc}
\hline Characteristic & OCTR & MECTR & T (P-value) \\
\hline Sex (male/female) & $16 / 30$ & $18 / 30$ & $0.144(0.850)$ \\
Age (years) & $48.0 \pm 5.33$ & $50.8 \pm 7.97$ & $2.159(0.170)$ \\
Affected hand (left/right) & $12 / 34$ & $16 / 32$ & $1.138(0.597)$ \\
Course of disease (months) & $10.6 \pm 2.86$ & $11.0 \pm 1.83$ & $0.196(0.507)$ \\
Operative time (min) & $31.5 \pm 3.3$ & $12.5 \pm 2.3$ & $2.276(<0.001)$ \\
Hospitalization time (days) & $5.5 \pm 1.5$ & $2.8 \pm 0.9$ & $6.844(<0.001)$ \\
Return to work/normal life (days) & $26.2 \pm 2.0$ & $11.6 \pm 2.2$ & $3.400(<0.001)$ \\
Follow-up duration (months) & $34.0 \pm 3.31$ & $33.3 \pm 3.13$ & $0.042(0.358)$
\end{tabular}

MECTR, modified endoscopic carpal tunnel release; OCTR, open carpal tunnel release.

Table III. Observation indexes compared between the two groups.

\begin{tabular}{lrrr}
\hline Characteristic & OCTR $(\mathrm{n}=46)$ & MECTR $(\mathrm{n}=48)$ & T $(\mathrm{P}-\mathrm{value})$ \\
\hline Grip strength $\left(\mathrm{g} / \mathrm{mm}^{2}\right)$ & $23.2 \pm 3.7$ & $23.3 \pm 1.9$ & $-0.039(0.969)$ \\
Pinch strength $\left(\mathrm{g} / \mathrm{mm}^{2}\right)$ & $6.3 \pm 1.6$ & $6.3 \pm 1.9$ & $0.021(0.983)$ \\
Two-point discrimination $(\mathrm{mm})$ & $6.8 \pm 1.4$ & $4.5 \pm 1.7$ & $0.217(0.829)$ \\
VAS (points) & $2.8 \pm 1.2$ & $2.1 \pm 0.9$ & $2.127(0.039)$ \\
Sympathetic dystrophy & $6(13.0)$ & $4(8.3)$ & $-0.514(0.610)$ \\
Kelly excellent or good & $36(78.3)$ & $42(87.5)$ & $0.831(0.411)$ \\
\hline
\end{tabular}

Values are expressed as the mean \pm standard deviation or $\mathrm{n}(\%)$. MECTR, modified endoscopic carpal tunnel release; OCTR, open carpal tunnel release; VAS, visual analogue scale.

thenar muscle atrophy and require functional revascularization. The remaining two groups of patients were revealed to exhibit no significant differences in the severity of the disease and Hamada grade $(\mathrm{P}>0.05$; Table I).

Patient follow up. All of the 94 patients were followed up for 3-41 months, with an average follow-up time of 33.6 months. In the OCTR group, one case exhibited incomplete remission of symptoms following the operation, with finger numbness reappearing after 3.5 months. The symptom was relieved after injection with triamcinolone acetonide and no recurrence was observed during the subsequent 6-month follow-up. The remaining 93 patients were reported to have completely recovered. The operative time $(12.5+2.3$ vs. $31.5+3.3 \mathrm{~min} ; \mathrm{P}<0.01)$, hospitalization time $(2.8+0.9$ vs. $5.5+1.5$ days; $\mathrm{P}<0.01)$ and time required to return to normal life (11.6 \pm 2.2 vs. $26.2 \pm 2.0$ days; $\mathrm{P}<0.01)$ in the MECTR group were significantly shorter compared with those in the OCTR group. The visual analogue scale score points, evaluating surgical area pain at 1 month following surgery, were lower in the MECTR group compared with those in the OCTR group $(2.1+0.9$ vs. $2.8+1.2 ; \mathrm{P}<0.01)$.

Other indicators, including excellent and good rates of Kelly grading ( 87.5 vs. $78.3 \%$; $\mathrm{P}>0.01)$ and the incidence of sympathetic dystrophy at 3 months following surgery (8.3 vs. $13.0 \%$; $>0.01$ ) were similar in the MECTR and OCTR groups. The incisions made in both groups were Class-A healing. The two-point discrimination ability of the index finger abdomen
( $<5 \mathrm{~mm}$ ) was recovered 1 week following surgery in both groups (Tables II and III).

\section{Discussion}

The CT is an inelastic fibro-osseous tunnel defined by the carpal bones and the flexor retinaculum. CTS may be caused by increased CT content (24-26) or decreased CT volume. Primary CTS results from the compression of the median nerve by the transverse carpal ligament (27). At present, the standard surgical treatment of CTS consists of an open incision and endoscopic release. Gurpinar et al (28) compared the clinical results and complications between OCTR and ECTR. In recent years, traditional open surgery has been gradually replaced by endoscopic release; this is due to the disadvantages of traditional open surgery, including the risk of serious injuries, a slow recovery time and post-operative recurrence caused by adhesion, intractable pain of the incision scar and other complications. It should be noted that due to the limited surgical field, nerve injury in the endoscopic group is more common compared with that in patients who receive open surgery (29). At present, the endoscopic treatment of CTS may be divided into two types: Those with a single incision as part of the Agee technique (30) and Okustu technique (31), and those with a double incision as part of the Chow technique (32). The single-incision Okustu technique uses a special facet endoscopic system, which is 
expensive and requires a special latch-up plastic casing. Therefore, the surgery requires to be performed outside the casing. Under these circumstances, the hook knife is easily interfered with by the surrounding soft tissue and may therefore deviate from the cannula when entering and operating along the outer wall of the tube, resulting in injuries to the blood vessels, nerve and flexor tendon, which are major causes of complications following surgery. For the double-incision Chow technique, the endoscope is placed in an opaque mantle tube, which forms a relatively narrow field of vision. In addition, the operation is vulnerable to the accumulation of subcutaneous fat and blood after incising the transverse carpal ligament; the operative steps are relatively complex and the learning curve to perform this technique is long $(33,34)$.

Compared with the single incision technique, an additional distal incision may increase the risk of collateral damage to the distal tissue. However, ECTR is currently, in our experience, only performed in large regional hospitals in China. This is firstly due to the fact that special assistive devices are required, including transparent cannulas, special push broaches and special facet endoscopic systems and the fact that grass-roots hospitals, even numerous Grade IIIA hospitals, frequently do not have the corresponding equipment and conditions. In addition, high levels of surgical expertise are required, as an improper operation may be prone to causing iatrogenic side injuries. Furthermore, it is difficult for beginners to master the operating essentials. Therefore, a long learning curve and related skills training are required.

Compared with previous studies on ECTR (35), the procedure reported in the present manuscript differs as the location of the incision and equipment required is altered. The present study made a single incision at the level of the proximal transverse carpal crease without the aid of special auxiliary endoscopic instruments. A common endoscopic lens (4.0-mm diameter, $30^{\circ}$ wide-angle arthroscope lens) was also used, and as the metal mantle tube was not inserted during the operation, the working diameter of the surgical instrument was markedly reduced. Therefore, the compression of the median nerve during endoscopic implantation was effectively reduced, resulting in a decreased incidence of post-operative sympathetic dystrophy (36). In addition, the fat suspension technique was used during this operation, which markedly increased the operation space. No median nerve injury occurred in the present study. The distal transverse carpal crease is the axial point of wrist movement; therefore, choosing it as the entrance allows the incision to be stimulated repeatedly in daily activities, leading to more obvious scars, redness and pain (37). In the present study, the surgical incision was moved from the distal to the proximal transverse carpal crease, which therefore avoided the abovementioned problem effectively. In addition, the incision may be appropriately expanded to increase the scope of the naked eye, the median nerve is well exposed and the risk of median nerve injury is further reduced by using soft glue tube and other protective measures. This method achieved a similar curative effect to that reported by others $(28,38)$.

As a developing country, China has an uneven distribution of medical care. The level of medical care in the majority of regions is relatively low and the medical equipment is relatively simple. With the method described in the present study, a special endoscopic system is no longer required; therefore, modified endoscopic surgery may be performed with the aid of an ordinary knee arthroscopic system, which is relatively low-cost and easy to obtain. In addition, the present method described may be easily learned, demonstrating a short learning curve. Therefore, the current method may be performed in areas with less advanced medical systems.

However, the present method also has several limitations; for instance, the lack of long palmar tendons in certain patients had a certain impact on the location of the incision. In addition, for Hamada Grade III patients and as the secondary surgery for patients with CTS, this operation may not be applicable.

In conclusion, the results of the present study suggested the use of MECTR as a novel endoscopic technology for CTS treatment. The MECTR technique exhibited several advantages, including a small risk of trauma, improved safety and effectiveness and feasibility of being performed in grass-roots hospitals with relative ease, which suggested that this method should be more widespread for the clinical treatment of CTS.

\section{Acknowledgements}

Not applicable.

\section{Funding}

The work was financed by a grant from Nanjing Science and Technology Development Plan Project in 2016 (grant no. 201605066) and Key Projects of the Science and Technology Development Fund of Nanjing Medical University in 2016 (grant no. 2016NJMUZD036).

\section{Availability of data and materials}

The datasets used and/or analyzed during the current study are available from the corresponding author on reasonable request.

\section{Authors' contributions}

ZC, JL, XXW and TBY were responsible for data acquisition and interpretation. ZC, DWC and XXW were responsible for formal data analysis. TBY and DWC carried out the investigation. ZC and DWC wrote the original draft. JQ designed this study and edited the manuscript. JQ and JL confirm the authenticity of the raw data. All authors read and approved the final manuscript.

\section{Ethics approval and consent to participate}

This study protocol was approved by the Ethics Committee of Sir Run Run Hospital, Nanjing Medical University (Nanjing, China) and was performed in accordance with the Declaration of Helsinki and institutional guidelines. Written informed consent was obtained from all individuals or their family members.

\section{Patient consent for publication}

The patients provided written informed consent for the publication of any associated data and accompanying images. 


\section{Competing interests}

The authors declare that they have no competing interests.

\section{References}

1. Sharief F, Kanmani J and Kumar S: Risk factors, symptom severity and functional status among patients with carpal tunnel syndrome. Neurol India 66: 743-746, 2018.

2. Gelfman R, Melton LJ, Yawn BP, Wollan PC, Amadio PC and Stevens JC: Long-term trends in carpal tunnel syndrome. Neurology 72: 33-41, 2009 .

3. Patterson JD and Simmons BP: Outcome assessment in carpel tunnel syndrome. Hand Clin 18: 359-363, 2002.

4. Akelman E and Weiss AP: Carpal tunnel syndrome. Etiology and endoscopic treatment. Orthop Clin North Am 26: 769-778, 1995.

5. Zyluk A and Strychar J: A comparison of two limited open techniques for carpal tunnel release. J Hand Surg Br 31: 466-472, 2006.

6. Kiymaz N, Cirak B, Tuncay I and Demir O: Comparing open surgery with endoscopic releasing in the treatment of carpal tunnel syndrome. Minim Invasive Neurosurg 45: 228-230, 2002.

7. Means KR, Parks BG, Lee SK and Segalman KA: Release of the transverse carpal ligament alone is associated with elevated pressure beneath the distal volar forearm fascia in a cadaver model of carpal tunnel syndrome. J Hand Surg Am 32: 1533-1537, 2007.

8. Papachristos A and Chow JCY: Endoscopic carpal tunnel release: Seventeen years' experience with the chow technique and specific anatomic considerations regarding the volar aspect of the wrist (SS-61). Arthroscopy 22: E31, 2006.

9. Uchiyama S, Yasutomi T, Fukuzawa T, Nakagawa $H$, Kamimura $\mathrm{M}$ and Kato $\mathrm{H}$ : Reducing neurologic and vascular complications of endoscopic carpal tunnel release using a modified chow technique. Arthroscopy 23: 816-821, 2007.

10. Kawee P, Pravit K and Adisorn P: Carpal tunnel release by mini palmar incision. Asian Biomed 5: 139-142, 2011.

11. Nagle DJ: Endoscopic carpal tunnel release. Hand Clin 18 307-313, 2002

12. Okutsu I, Hamanaka I, Chiyokura Y, Miyauchi Y and Sugiyama K: Intraneural median nerve pressure in carpal tunnel syndrome. J Hand Surg Br 26: 155-156, 2001.

13. Sayegh ET and Strauch RJ: Open versus endoscopic carpal tunnel release: A meta-analysis of randomized controlled trials. Clin Orthop Relat Res 473: 1120-1132, 2015.

14. Trumble TE, Diao E, Abrams RA and Gilbert-Anderson MM: Single portal endoscopic carpal tunnel release compared with open release: A prospective, randomized trial. J Bone Joint Surg Am 84: 1107-1115, 2002.

15. Graham B, Regehr G, Naglie G and Wright JG: Development and validation of diagnostic criteria for carpal tunnel syndrome. J Hand Surg Am 31: 919-924, 2006.

16. Gilliatt RW and Meer J: The refractory period of transmission in patients with carpal tunnel syndrome. Muscle Nerve 13: 445-450, 1990.

17. Atroshi I, Gummesson C, Johnsson R, Ornstein E and Rosén I: Median nerve latency measurement agreement between portable and conventional methods. J Hand Surg Br 25: 73-77, 2000.

18. Shinoda J, Hashizume H, McCown C, Senda M, Nishida K, Doi $\mathrm{T}$ and Inoue $\mathrm{H}$ : Carpal tunnel syndrome grading system in rheumatoid arthritis. J Orthop Sci 7: 188-193, 2002.

19. Macdermid JC, Richards RS, Roth JH, Ross DC and King GJ: Endoscopic versus open carpal tunnel release: A randomized trial. J Hand Surg Am 28: 475-480, 2003.
20. Wewers ME and Lowe NK: A critical review of visual analogue scales in the measurement of clinical phenomena. Res Nurs Health 13: 227-236, 1990.

21. Ciccone W II: Editorial Commentary: What's Hamada with partial rotator cuff repair? Arthroscopy 35: 351-352, 2019.

22. Takayama K, Yamada S and Kobori Y: Clinical effectiveness of mini open superior capsular reconstruction using autologous tensor fascia lata graft. J Shoulder Elbow Surg: Sep 30, 2020 (Epub ahead of print). doi: 10.1016/j.jse.2020.09.005.

23. Hamada K, Fukuda H, Mikasa M and Kobayashi Y: Roentgenographic findings in massive rotator cuff tears. A long-term observation. Clin Orthop Relat Res: 92-96, 1990.

24. Zamora CA, Zamora MA, Soto JD and Garcés MA Myoepithelioma of the hand and carpal tunnel: An unusual cause of median nerve compression. J Clin Ultrasound 39: 44-47, 2010

25. Mohamed A, Rosalie S, Taylor K, Fink M, Coombs C, Ryan M and Kornberg A: Carpal tunnel syndrome secondary to ganglion cyst in a child. J Child Neurol 26: 630-633, 2011.

26. Robinson AJ, Haj Basheer M and Herbert K: An unusual cause of carpal tunnel syndrome. J Plast Reconstr Aesthet Surg 63: e788-e791, 2010

27. Yücetaş SC and Yildirim A: Comparative results of standard open and mini open, knifelight instrument-assisted carpal tunnel release. J Neurol Surg A Cent Eur Neurosurg 74: 392-399, 2013.

28. Gurpinar T, Polat B, Polat AE, Carkci E, Kalyenci AS and Ozturkmen Y: Comparison of open and endoscopic carpal tunnel surgery regarding clinical outcomes, complication and return to daily life: A prospective comparative study. Pak J Med Sci 35: $1532-1537,2019$

29. Trehan SK, Lyman S, Ge Y, Do HT and Daluiski A: Incidence of nerve repair following endoscopic carpal tunnel release is higher compared to open release in New York State. HSS J 15: 143-146, 2019.

30. Worseg AP, Kuzbari R, Korak K, Höcker K, Wiederer C, Tschabitscher M and Holle J: Endoscopic carpal tunnel release using a single-portal system. Brit J Plast Surg 49: 1-10, 1996.

31. Okutsu I, Ninomiya S, Takatori Y and Ugawa Y: Endoscopic management of carpal tunnel syndrome. Arthroscopy 5: 11-18, 1989.

32. Chow JC and Hantes ME: Endoscopic carpal tunnel release: Thirteen years' experience with the Chow technique. J Hand Surg Am 27: 1011-1018, 2002.

33. Chow JC: Endoscopic release of the carpal ligament for carpal tunnel syndrome: 22-month clinical result. Arthroscopy 6: 288-296, 1990

34. Okutsu I, Ninomiya S, Natsuyama M, Takatori Y, Inanami H, Kuroshima N and Hiraki S: Subcutaneous operation and examination under nuiversal endoscope. Nihon Seikeigeka Gakkai Zasshi 61: 491-498, 1987 (In Japanese).

35. Hansen TB and Majeed HG: Endoscopic carpal tunnel release. Hand Clin 30: 47-53, 2014.

36. Tuzuner S, Sherman GM, Ozkaynak S and Ozcanli H: Endoscopic carpal tunnel release: Modification of Menon's technique and data from 191 cases. Arthroscopy 20: 721-727, 2004.

37. Agee JM, Peimer CA, Pyrek JD and Walsh WE: Endoscopic carpal tunnel release: A prospective study of complications and surgical experience. J Hand Surg Am 20: 165-172, 1995.

38. Michelotti B, Romanowsky D and Hauck RM: Prospective, randomized evaluation of endoscopic versus open carpal tunnel release in bilateral carpal tunnel syndrome: An interim analysis. Ann Plast Surg 73 (Suppl 2): S157-S160, 2014.

(i) 9 This work is licensed under a Creative Commons cc) $\mathrm{EY}$ NO ND Attribution-NonCommercial-NoDerivatives 4.0 International (CC BY-NC-ND 4.0) License. 